Irish Section Conference, 22-24 June 2021, Nutrition, health and ageing — translating science into practice - Part A

\title{
Mycoprotein reduces endogenous glucose production when consumed with a mixed-meal tolerance test
}

\author{
G. Whelehan ${ }^{1}$, S. West ${ }^{1}$, T.J.A. Finnigan ${ }^{2}$, B.T. Wall ${ }^{1}$ and F.B. Stephens ${ }^{1}$ \\ ${ }^{1}$ Department of Sport and Health Sciences, University of Exeter, Exeter, UK and \\ ${ }^{2}$ Marlow Foods Ltd, Stokesley, UK
}

Postprandial glucose kinetics can be managed by altering dietary composition. The addition of soluble fibre to a meal can decrease the total rate of glucose appearance $(\mathrm{RaT})^{(1)}$, while insoluble fibre has been shown to increase the rate of glucose disappearance $(\mathrm{RdT})^{(2)}$. Mycoprotein (MYC) is a high-fibre food (1/3 soluble and 2/3 insoluble) shown to reduce postprandial glucose and insulin concentrations in healthy participants ${ }^{(3)}$, though the underlying glucose kinetics have not been explored. The present study took a dual stable isotope tracer approach to determine how MYC impacts glucose kinetics when ingested with a mixed-meal. We hypothesised that the previously observed reduction in glucose concentrations with MYC ingestion would be due to a lower RaT. This study also aimed to determine whether this effect was dose dependent.

In a double-blind, randomised, cross-over design, 12 healthy adults (M:F 6:6) attended 3 experimental test days, involving ingestion of a test drink, enriched with $100 \mathrm{mg}$ [U-13C] glucose, containing $250 \mathrm{ml}$ whole milk, $50 \mathrm{~g}$ glucose, and either 0 (CON), 20 (MYC20), or $40 \mathrm{~g}$ (MYC40) MYC. CON and MYC20 were matched for energy, protein (18 g), carbohydrate (75 g) and fat (12 g). An intravenous infusion of D-[6,6-2H2] glucose was used to determine glucose kinetics over $6 \mathrm{~h}$. Time-course and AUCs of glucose, insulin, and rate of appearance of total (RaT), exogenous (RaEx), endogenous (EGP), and RdT, of glucose were assessed using two- and one-way ANOVAs, respectively.

Drink ingestion resulted in a rapid increase in blood glucose and serum insulin concentrations, peaking at 45 and 30 min, respectively; however there were no differences between the conditions $(P>0.05)$. Both RaT and RdT decreased in the MYC40 compared with CON during $0-120 \mathrm{~min}(-14 \pm$ SEM $4 \%$ and $-16 \pm 5 \%$, respectively) $(P<0.05)$. RaEx was not affected by MYC in the first $0-120$ min, but during 240-360 min RaEx was 14 ( \pm 10$) \%$ greater in MYC40 than CON. MYC20 and MYC40 suppressed EGP by $19 \pm 7 \%$ and $14 \pm 5 \%$ respectively, compared to CON between 0-120 min, and this effect continued into 120-240 min for MYC20 but not for MYC40.

The present study showed MYC ingestion reduced postprandial EGP, implying that MYC, (or a product of its metabolism) directly affects the liver, particularly given there was no difference between MYC and CON in circulating insulin concentration.

\section{References}

1. Boers HM, van Dijk TH, Himestra H, et al. (2017) Br J Nutr 118, 777-787.

2. Robertson MD, Currie JM, Morgan LM, et al. (2003) Diabetologia 46, 659-665.

3. Turnbull WH \& Ward T (1993) Am J Clin Nutr 61, 135-40. 